\title{
Very-short term solar power generation forecasting based on trend-additive and seasonal-multiplicative smoothing methodology
}

\author{
Stanislav Eroshenko ${ }^{1}$, Alexandra Khalyasmaa ${ }^{1, *}$ and Rustam Valiev ${ }^{1}$ \\ ${ }^{1}$ Ural Federal University named after the first President of Russia B.N. Yeltsin, Ekaterinburg, 620002 Mira str. 19, Russia
}

\begin{abstract}
In conditions of development of generating facilities on renewable energy sources, the technology runs up to uncertainty in the operational and short-term planning of the power system operating modes. To date, reliable tools for forecasting the generation of solar power stations are required. This paper considers the methodology of operational forecasting of solar power stations output based on the mathematical apparatus of cubic exponential smoothing with trend and seasonal components. The presented methodology was tested based on the measuring data of a real solar power station. The average forecast error was not more than $10 \%$ for days with variable clouds and not more than 3\% for clear days, which indicates the effectiveness of the proposed approach.
\end{abstract}

\section{Introduction}

In conditions of the energy market development, the role of effective management tasks at all levels - the power station, the power system, the power pool system - is immeasurably increasing. At the same time, the effectiveness of the operational optimal control of the power system mode is firstly determined by the correctness and accuracy of the forecast for the power stations generation and the load power. The most important procedures in the power joint market are the competitive capacity auction on the day-ahead market, solving the technical and economic problem to select the live generating equipment, as well as the calculation and considering generation patterns under the power system actual control on-line within the balancing power market.

In accordance with the rules for the price bids submission by participants in the wholesale market [1], the applications of electricity suppliers are submitted taking into account following requirements:

- applications on the day-ahead market are sent for every hour of the day $X$ (applications are sent per day $X-1$ to 13:30 Moscow time);

- applications on the balancing market are sent every hour (one hour before calculating the balancing market plan).

In conditions of development of generating facilities on renewable energy sources, the degree of uncertainty in the operational and short-term planning of the power system operating modes is significantly increased. To date, reliable tools for forecasting the generation of power stations using, in particular, solar energy are required. Taking into account the nature of the wholesale electricity market in the UES of Russia, the short-term forecasting of solar power stations generation is considered in terms of the application on the day-ahead market. It is also required to obtain reference data on the solar power station generation for the day $X+1, X+2, \ldots$, $X+N$, depending on the availability of relevant weather forecast data provided by the weather provider.

When controlling the operating mode of the power system on-line, it is necessary to compensate the departure from the mode planned one day ago. Therefore, the task of operational forecasting is considered in terms of filing applications on a balancing market for the next hour, as well as for obtaining reference data on the solar power station generation for several hours ahead.

To date, there are many methods for forecasting the generation of solar power stations based on the forecasting the solar radiation flux density. There are a number of outstanding methodologies based on the identification of satellites images of cloud movement in the area where the object under consideration is located [2].

In a number of works, it is proposed to use specialized measuring instruments that allow quickly assessing the weather conditions. The use of these methods, as a rule, is limited by the characteristics of the weather data being analyzed, or requires additional investments for the installation of meteorological measuring posts. From the point of view of implementation, statistical methods of time series analysis, such as moving average method, autoregressive models, etc. are the most simple $[3,4]$. In this paper, the application of exponential smoothing methods to solve the problem of solar power stations operational forecasting is considered.

For small intervals from 1 to 3 hours, the generation forecast can be significantly improved by using current

\footnotetext{
*Corresponding author: 1khalyasmaa@mail.ru
} 
data obtained by direct observation methods. The main requirement for measuring current weather conditions at a solar power station is the horizontally installed pyranometer for measuring the total flux density of solar radiation. Current measurements obtained from the pyranometer are used in the algorithm of operational forecasting of solar power stations.

The accuracy of the forecast, as a rule, is estimated by the deviations between the forecast and actual values; the following attributes are often used to estimate accuracy [5]:

- mean absolute error value - is useful in assessing the possible penalties associated with the forecast inaccuracy;

- mean-square error value - more focuses on large departures of the forecast from actual values;

- systematic error values associated with underestimation or revaluation of output.

\section{Methodology description}

The operational forecast is implemented to control the operation of the solar power station (5-120 minutes) and is actually a refinement of the shortterm forecast adjusted for current measurement data. Forecasting for relatively small prediction intervals is effectively implemented based on statistical methods of time series analysis, in particular, the exponential smoothing method [6].

Exponential smoothing is one of the most common methods of leveling the series. It is based on the calculation of exponential averages. Exponential smoothing of the series is carried out according to the recurrence formula:

$$
S_{t}=\alpha \cdot y_{t-1}+(1-\alpha) \cdot S_{t-1},
$$

where $S_{t}$ is the exponential average at the time $t$; $\alpha$ is the smoothing parameter ( $\alpha=$ const, $0<\alpha<1$ ); $y_{t-1}$ is the actual value of the solar radiation flux density at the time $t-1 ; S_{t-1}$ is the exponential average at the time $t-1$.

If the recurrence relation (1) is used successively, the exponential average $S_{t}$ can be expressed in terms of the values of the series $y$ :

$$
S_{t}=\alpha \sum_{i=0}^{N-1}(1-\alpha)^{i} y_{t-i}+\beta_{N} S_{0}
$$

where $S_{t}$ is the exponential average at the time $t$; $N$ is the number of series terms; $\alpha$ is the smoothing parameter ( $\alpha=$ const, $0<\alpha<1) ; y_{t-i}$ is the actual value of the solar radiation flux density at the time $t-i ; \beta_{N}$ is the parameter for smoothing the initial conditions; $S_{0}$ is the value of the solar radiation flux density, which characterizes the initial conditions for the first application of formula (1) for $t=0$.
As the initial approximation, the value $S_{t}$ turns out to be the weighted sum of all the terms of a series consisting of average values of the solar radiation flux density, and the weights decrease exponentially as a function of the prescription ("age") of the observation.

Exponential alignment always requires a previous exponential average. When the process is beginning, there must be some value $S_{0}$ that can be used as the value preceding $S_{1}$. Thus, if there are past data on the flux density of solar radiation by the time of the beginning of the alignment process, then the arithmetic mean of all available points or some part of them can be used as an initial value $S_{0}$.

Identification of the initial level of the series $S_{0}$ can also be performed based on a priori knowledge about the process of changing the flux density of solar radiation during the day or based on its analogy with other processes. After $k$ steps, the weight given to the initial value is $(1-\alpha)^{k}$. If there is confidence in the validity of the initial value $S_{0}$, then the coefficient $\alpha$ can be taken as small. If there is no such confidence, the parameter $\alpha$ should be given a large value, so that the influence of the initial value decreases rapidly. However, a large value of $\alpha$ can cause a large dispersion of $S_{t}$ oscillation. If suppression of these oscillations is required, then after sufficient removal from the initial time moment, the value $\alpha$ can be reduced.

The described approach is an exponential smoothing of the first order, which is mainly applicable for simple data without pronounced trends or seasonal changes, but when the data includes these components, it is recommended to use exponential smoothing of the second and third degree for more accurate forecast. Since the value predicted in the work described - the flux density of solar radiation - has seasonals, it is recommended to use cubic exponential smoothing. Basic equations for its implementation are presented below:

$$
P_{f(t)}=\alpha \cdot \frac{P_{t-1}}{I_{t-L}}+(1-\alpha) \cdot\left(P_{f(t-1)}+b_{t-1}\right),
$$

where $P_{f(t)}$ is the exponential average at the time $t$; $\alpha$ is the smoothing parameter ( $\alpha=$ const, $0<\alpha<1$ ); $P_{t-1}$ is the actual value of the solar radiation flux density at the time $t-1 ; b_{t-1}$ is the trend component at time $t-1 ; I_{t-L}$ is the indicator that takes into account seasonals in the flux density of solar radiation.

The trend component for the time $t$ is determined by the formula: 


$$
b_{t}=\gamma\left(P_{f(t)}-P_{f(t-1)}\right)+(1-\gamma) b_{t-1},
$$

where $b_{t}$ is the trend component at time $t ; \gamma$ is the smoothing parameter ( $\gamma=$ const, $0<\gamma<1) ; P_{f(t)}$ is the exponential average at the time $t ; P_{f(t-1)}$ is the exponential average at the time $t-1$; is the trend component at time $t-1$.

The indicator taking into account seasonals of the predicted value is determined by the formula:

$$
I_{t}=\beta \cdot \frac{P_{t}}{P_{f(t)}}+(1-\beta) \cdot I_{t-L} \cdot \gamma,
$$

where $I_{t}$ is the indicator taking into account seasonals of the predicted value for the current season; $\beta \quad$ is the smoothing parameter ( $\beta=$ const, $0<\beta<1$ ); $P_{t}$ is the actual value of the predicted value at the time $t ; P_{f(t)}$ is the exponential average at the time $t ; I_{t-L}$ is the indicator taking into account seasonals of the predicted value for the previous season; $\gamma$ is the smoothing parameter $(\gamma=$ const, $0<\gamma<1)$.

It is possible to determine the predicted value for $m$ intervals ahead based on the trend component for the time $t$ and the indicator that takes into account seasonals of the predicted value:

$$
P_{f(t) t+m}=\left(P_{f(t)}+m \cdot b_{t}\right) \cdot I_{t-L+m},
$$

where $P_{f(t) t+m}$ is the predicted value for $m$ intervals ahead; $P_{f(t)}$ is the exponential average at the time $t$; $b_{t}$ is the trend component at time $t ; I_{t-L+m}$ is the indicator taking into account seasonals of the predicted value within the season for $m$ intervals ahead.

To use the model, initialization of the parameters at the initial stage is necessary. If the forecasted data includes $N$ periods, then in order to estimate the trend component it is recommended to use the initial retrospective data of at least $2 N$ periods. The equation for the initial estimate of the indicator $b$ is as following:

$$
b=\frac{1}{N}\left(\frac{P_{N+1}-P_{1}}{N}+\frac{P_{N+2}-P_{2}}{N}+\ldots+\frac{P_{N+N}-P_{N}}{N}\right)
$$

It is also necessary to calculate the initial characteristics of the seasonals effect. Assume that there are data on the solar radiation flux density for 6 years, with data for 4 seasons for each year. At the first stage, the average value of the predicted parameter is calculated for each year $y$ :

$$
A_{y}=\frac{\sum_{i=1}^{4} P_{i}}{4}, y=1,2,3 \cdots 6
$$

At the second stage, the specific weights of the influence of each season for each year are estimated by dividing the actual values of the predicted parameter by the average for each year.

$\begin{array}{cccccc}1 & 2 & 3 & 4 & 5 & 6 \\ P_{1} / A_{1} & P_{5} / A_{2} & P_{9} / A_{3} & P_{13} / A_{4} & P_{17} / A_{5} & P_{21} / A_{6} \\ P_{2} / A_{1} & P_{6} / A_{2} & P_{10} / A_{3} & P_{14} / A_{4} & P_{18} / A_{5} & P_{22} / A_{6} \\ P_{3} / A_{1} & P_{7} / A_{2} & P_{11} / A_{3} & P_{15} / A_{4} & P_{19} / A_{5} & P_{23} / A_{6} \\ P_{4} / A_{1} & P_{8} / A_{2} & P_{12} / A_{3} & P_{16} / A_{4} & P_{20} / A_{5} & P_{24} / A_{6}\end{array}$

The obtained results are used in the third stage to find seasonal coefficients as the arithmetic mean of the calculated values.

$$
\begin{aligned}
& I_{1}=\frac{P_{1} / A_{1}+P_{5} / A_{2}+P_{9} / A_{3}+P_{13} / A_{4}+P_{17} / A_{5}+P_{21} / A_{6}}{6} \\
& I_{2}=\frac{P_{2} / A_{1}+P_{6} / A_{2}+P_{10} / A_{3}+P_{14} / A_{4}+P_{18} / A_{5}+P_{22} / A_{6}}{6} \\
& I_{3}=\frac{P_{3} / A_{1}+P_{7} / A_{2}+P_{11} / A_{3}+P_{15} / A_{4}+P_{19} / A_{5}+P_{23} / A_{6}}{6} \\
& I_{4}=\frac{P_{4} / A_{1}+P_{8} / A_{2}+P_{12} / A_{3}+P_{16} / A_{4}+P_{20} / A_{5}+P_{24} / A_{6}}{6}
\end{aligned}
$$

The special attention should be paid to choosing the value of smoothing constants $\alpha, \beta$ and $\gamma$. As a rule, these coefficients tend to be fit in such a way as to minimize the mean square deviation of the forecast from the actual measurements. The use of the presented model requires the availability of retrospective data, while reliable estimation of seasonals and trend components requires information on the predicted parameters for at least two seasons.

The exponential smoothing method Implementation for operational forecasting of solar power stations generation is possible with the following:

1. The development of the cubic exponential smoothing model taking into account the trend component and seasonals of the solar radiation flux density in the first stages of implementation can be limited by a model of the first or second order by the moment of accumulation of retrospective data on solar radiation flux density.

2. In order to ensure a unified approach to the forecasting problem, the function of calculating the operational forecast is expedient to be implemented for estimating the forecast of the solar radiation flux density. In this case, the solar power station generation can be found due to a known number and passport data of photovoltaic panels, inverters, converters and primary equipment on alternating current.

3. Taking into account the stochastic nature of the considered process and the presence of large "drops" in the time series of the solar flux density during periods of variable clouds in order to ensure the exponential smoothing method adaptability to the current design conditions, smoothing coefficients $\alpha, \beta$ and $\gamma$ will be found 
automatically for each current design day $X$ based on the previously calculated short-term forecast of the solar radiation flux density.

Input data: short-term forecast of the solar radiation flux density for the current day $X$ taking into account the updated weather forecast provided by the weather provider $\left\{\widehat{G}_{h 0}, \widehat{G}_{h 1}, \ldots \widehat{G}_{h 24}\right\}$.

Output data: an operational correction of the forecast of the solar flux density for the current day X taking into account the weather data received from the meteorological station established on the territory of the solar power station $\left\{G_{h 0}, G_{h 1}, \ldots, G_{h t}, \widehat{G}_{h t+1 \ldots}, \widehat{G}_{h 24}\right\}$.

Fitting the coefficients of the exponential smoothing function $\alpha, \beta$ and $\gamma$ is carried out till the actual sunrise time of the current day, based on the data of the short-term forecast of the solar flux density for the current day by estimating the weighted mean percentage error:

$$
W M P E=\frac{1}{N} \sum_{t=1}^{N} \frac{\left|\bar{G}^{m}(t)-\bar{G}^{f}(t)\right|}{\bar{G}_{0}(t)} \cdot 100 \%,
$$

where $\bar{G}^{m}(t)$ is the average measured value of the solar radiation flux density near the ground within the defined time step on the time interval $t,\left[\mathrm{~W} / \mathrm{m}^{2}\right] ; \bar{G}^{f}(t)$ is the average forecast value of the solar radiation flux density near the ground within the defined time step on the time interval $t,\left[\mathrm{~W} / \mathrm{m}^{2}\right] ; \bar{G}_{0}(t)$ is the solar radiation flux density at the boundary of the atmosphere for the defined time step $t,\left[\mathrm{~W} / \mathrm{m}^{2}\right]$. The flux density of solar radiation at the boundary of the atmosphere is determined in accordance with the expression [7]:

$$
\begin{gathered}
\bar{G}_{0}=\frac{12}{\pi} G_{\text {on }}\left(\cos \varphi \cdot \cos \delta \cdot\left(\sin \omega_{2}-\sin \omega_{1}\right)+\right. \\
\left.+\frac{\pi\left(\omega_{2}-\omega_{1}\right)}{180^{\circ}} \cdot \sin \varphi \cdot \sin \delta\right),
\end{gathered}
$$

where $\bar{G}_{0}$ is the average value of the solar radiation flux density at the boundary of the atmosphere within the defined time step, $\left[\mathrm{W} / \mathrm{m}^{2}\right] ; G_{o n}$ is the solar radiation flux density at the boundary of the atmosphere, $\left[\mathrm{W} / \mathrm{m}^{2}\right] ; \varphi$ is the latitude, [degrees]; $\delta$ is the solar declination angle, [degrees]; $\omega_{1}$ is the hour angle at the beginning of the interval, [degrees]; $\omega_{2}$ is the hour angle at the end of the interval, [degrees].

The weighting of the error at the boundary of the atmosphere will allow estimating the error relative to the conditionally constant "base" and showing how many percent the error is from the maximum possible flux density of solar radiation for the considered time interval [8].

This criterion is resistant to individual "spikes" of the model and provides the ability to fit automatically optimal smoothing coefficients for the current design day $X$ by the brute force, which is easily implemented programmatically.

\section{Calculation example}

Within the framework of the presented work, the feasibility of implementing the operational forecast function for the existing solar power station was analyzed. Geographic parameters, as well as the time zone parameters are presented in the Table 1.

Table 1. Geographic parameters of the solar power station location

\begin{tabular}{|c|c|c|c|c|}
\hline № & Parameter & Symbol & Units & Value \\
\hline 1. & Latitude & $b_{1}$ & [degrees] & 51.26 \\
\hline 2. & Longitude & $c_{1}$ & [degrees] & 58.47 \\
\hline 3. & $\begin{array}{c}\text { Difference with } \\
\text { Greenwich time }\end{array}$ & $d_{1}$ & [hours] & 5 \\
\hline
\end{tabular}

The data of current measurements for the period from 01.05.2017 to 28.05.2017 were taken for the analysis. Measuring data on the flux density of solar radiation is represented by averaged half-hour values. Since within the balancing market the information is submitted 1 hour before the planning of the power system, the forecasting of the solar power stations generation is 1 and 2 steps ahead. Their averaged value is taken as their predicted value.

The forecast error parameters for the characteristic days of the period under consideration are presented in Table 2 .

Table 2. The forecast error in case of cubic exponential smoothing

\begin{tabular}{|c|c|c|c|c|}
\hline № & Date & $\begin{array}{c}\text { Weather } \\
\text { conditions }\end{array}$ & $\begin{array}{c}\text { Weighted } \\
\text { error for } \\
\text { 30 minutes } \\
\text { ahead, \% }\end{array}$ & $\begin{array}{c}\text { Weighted } \\
\text { error for } \text { an } \\
\text { hour } \\
\text { ahead, \% }\end{array}$ \\
\hline 1. & 01.05 .17 & clear (no clouds) & 2.77 & 4.03 \\
\hline 2. & 03.05 .17 & light clouds & 4.36 & 5.52 \\
\hline 3. & 19.05 .17 & variable clouds & 7.35 & 9.49 \\
\hline 4. & 11.05 .17 & $\begin{array}{c}\text { continuous } \\
\text { clouds }\end{array}$ & 5.98 & 7.16 \\
\hline 5. & \multicolumn{2}{|c|}{$01.05 .2017-28.05 .2017$} & $\begin{array}{c}4.96 \\
\mathrm{~min}-1.87 \\
\mathrm{max}-9.85\end{array}$ & $\begin{array}{c}6.50 \\
\mathrm{~min}-2.45 \\
\mathrm{max}-12.69\end{array}$ \\
\hline
\end{tabular}

Graphical representation of the actual and forecast curve for some characteristic days of the period under consideration is presented in Figures 1-2.

To illustrate the effectiveness of the methods and approaches for operational forecasting of solar power generation, Figure 3 presents a graphical comparison of the accuracy of the short-term solar power station generation forecast for the day ahead and operational forecast. 


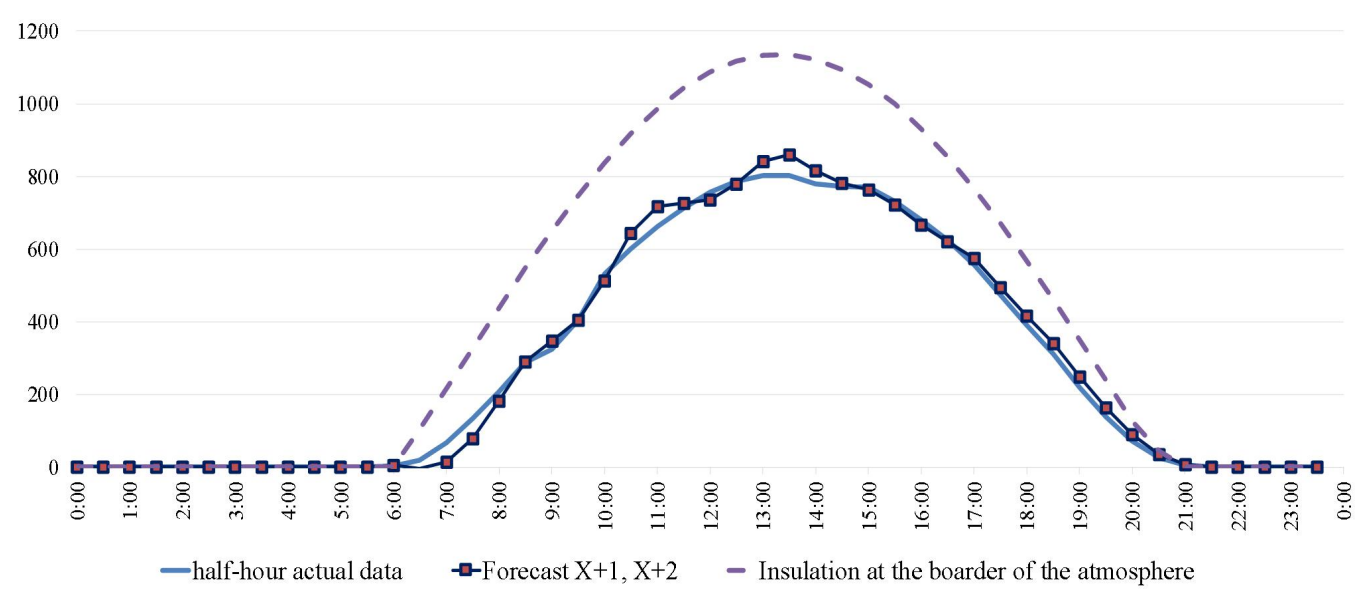

Figure 1. Hour-ahead exponential smoothing forecasting, 01.05.2017

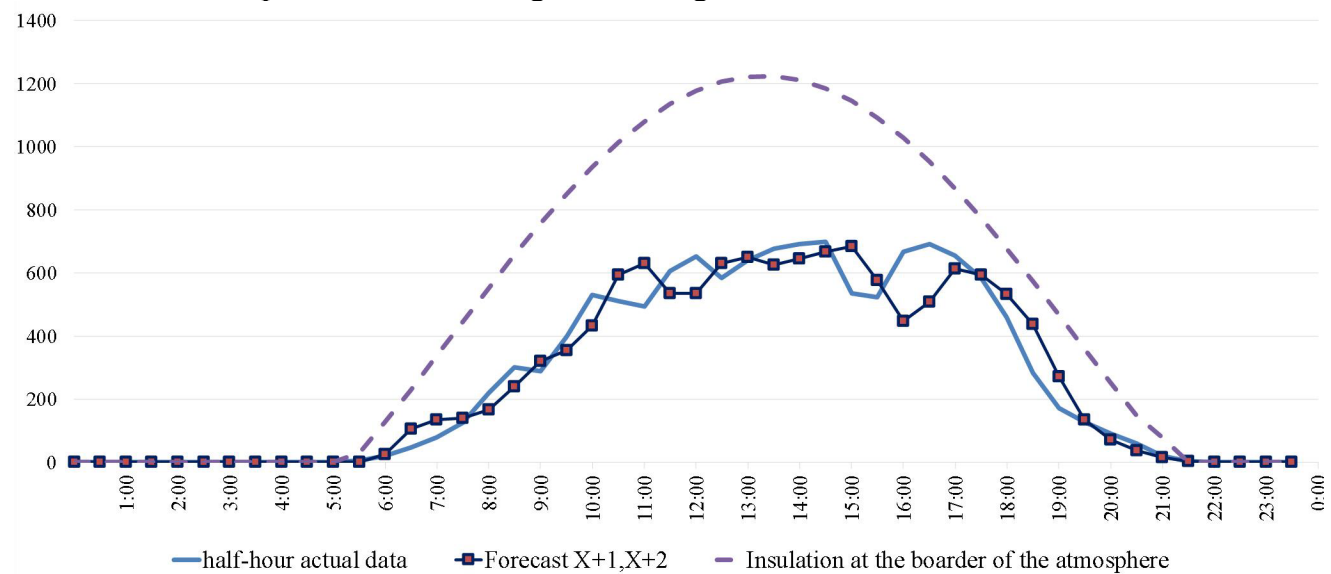

Figure 2. Hour-ahead exponential smoothing forecasting, 19.05.2017

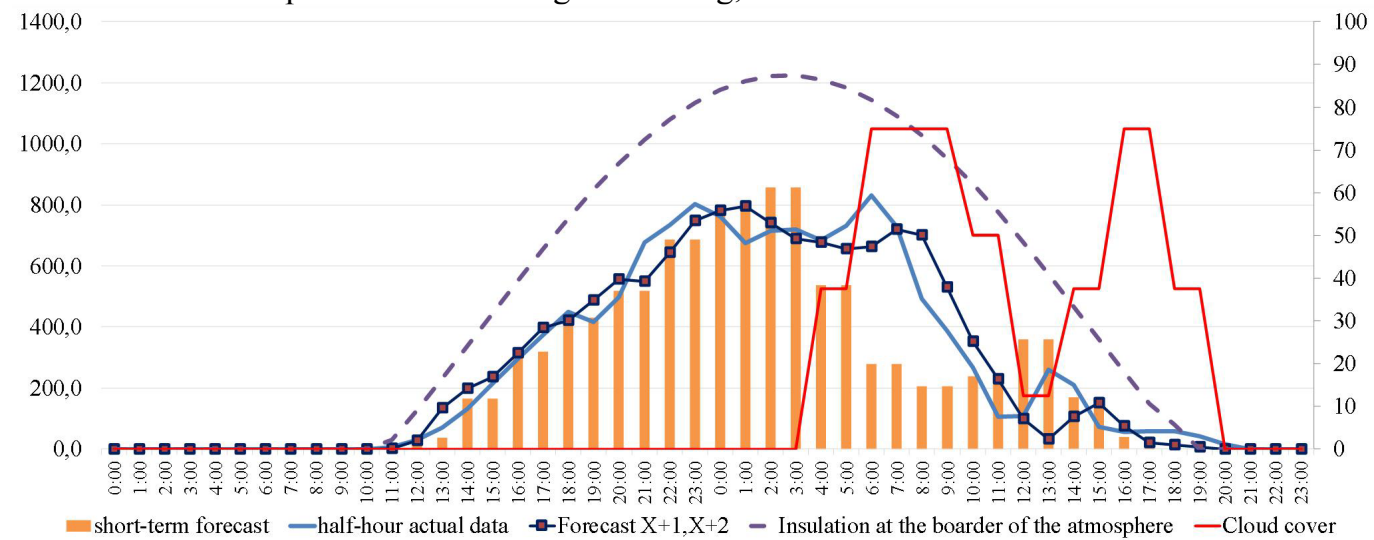

Figure 3. Hour-ahead exponential smoothing forecasting, 28.05.2017

Obviously, an operational correction based on current measurements allows to significantly reduce the error, which is due, among other things, to the low accuracy of weather data, on the basis of which the day ahead forecast was made.

\section{Conclusion}

In this paper an approach to operational forecasting of solar power generation based on the cubic exponential smoothing method, taking into account the trend and seasonal components in the initial sample, was considered.

Accounting the seasonal component, obtained by the methodology presented in this article, allows to significantly reduce the "backlog" effect typical for all forecasting methods based on the retrospective data analysis, thereby accuracy of forecasting on clear and cloudy days significantly increases.

The average error calculated with respect to the flux density of solar radiation at the boundary of the atmosphere was no more than $10 \%$, which proves the high accuracy of the forecast of output of the generating object. It should be noted that the error substantially 
increases as the forecast horizon deepens, which indicates the necessity to revise this methodology when predicting 3 or more hours ahead.

Greater accuracy can be achieved by increasing the discreteness of the information analyzed. Therefore, when predicting with a discreteness of 30 minutes, it is expedient to monitor the dynamics of the predicted parameter at 10-15 minutes intervals, which allows to correct the trend component promptly.

\section{References}

1. V. Margaret, J. Jose. Exponential Smoothing Models for Prediction of Solar Irradiance. International Journal of Advanced Research in Electrical, Electronics and Instrumentation Engineering (An ISO 3297: 2007 Certified Organization) Vol. 4, Issue 2, February 2015.

2. D.A. Snegirev, S.A. Eroshenko, R.T. Valiev, A.I. Khalyasmaa. Algorithmic realization of short-term solar power plant output forecasting. Proceedings of 2017 IEEE 2nd International Conference on Control in Technical Systems, CTS 2017. Pp. 228-231.

3. C. Severiano, F. G. Guimarães and M. W. Cohen, "Very short-term solar forecasting using multi-agent system based on Extreme Learning Machines and data clustering," 2016 IEEE Symposium Series on Computational Intelligence (SSCI), Athens, 2016, pp. $1-8$.

4. C.A. Severiano, P. C. L. Silva, H. J. Sadaei and F. G. Guimarães, "Very short-term solar forecasting using fuzzy time series," 2017 IEEE International Conference on Fuzzy Systems (FUZZ-IEEE), Naples, 2017, pp. 1-6.

5. A.D. Orjuela-Cañón, J. Hernández and C. R. Rivero, "Very short term forecasting in global solar irradiance using linear and nonlinear models," 2017 IEEE Workshop on Power Electronics and Power Quality Applications (PEPQA), Bogota, 2017, pp. 15.

6. S. Gupta, N. A. Shrivastava, A. Khosravi and B. K. Panigrahi, "Wind ramp event prediction with parallelized gradient boosted regression trees," 2016 International Joint Conference on Neural Networks (IJCNN), Vancouver, BC, 2016, pp. 5296-5301.

7. W. Glassley, J. Kleissl. Current state of the art in solar forecasting. California Renewable Energy Collaborative Final Report. 2013.

8. V. Prema. K. Uma Rao. Development of statistical time series models for solar power prediction. Renewable energy. 2015. 